\title{
Análise conjunta de atributos físicos e biológicos do solo sob sistema de integração lavoura-pecuária
}

\author{
Rogério Ferreira da Silva( ${ }^{(1)}$, Maria de Fátima Guimarães ${ }^{(2)}$, Adriana Maria de Aquino( ${ }^{(3)}$ e Fábio Martins Mercante ${ }^{(4)}$ \\ (1)Universidade Estadual de Mato Grosso do Sul, Rua Projetada A, s/no, CEP 79730-000 Glória de Dourados, MS. E-mail: rogerio@uems.br \\ (2)Universidade Estadual de Londrina, Centro de Ciências Agrárias, Caixa Postal 6001, CEP 86051-990 Londrina, PR. E-mail: mfatima@uel.br \\ (3)Embrapa Agrobiologia, Rodovia BR 465, Km 7, CEP 23851-970 Seropédica, RJ. E-mail: adriana@cnpab.embrapa.br (4)EmbrapaAgropecuária \\ Oeste, Caixa Postal 661, CEP 79804-970 Dourados, MS. E-mail: mercante@cpao.embrapa.br
}

\begin{abstract}
Resumo - O objetivo deste trabalho foi avaliar os efeitos do sistema integração lavoura-pecuária (ILP) sobre os atributos morfológicos, físicos e biológicos do solo. O experimento foi realizado em Dourados, MS, em Latossolo Vermelho distroférrico típico. Foram avaliados os seguintes sistemas: monocultura com sistema convencional de preparo do solo, ILP com sistema plantio direto, pastagem contínua de Urochloa decumbens (Syn. Brachiaria decumbens) e vegetação nativa. Em cada sistema, foi aberta uma trincheira com o intuito de detectar as modificações morfológicas provocadas pelos diferentes manejos com uso do método do perfil cultural. Os macroinvertebrados do solo foram avaliados conforme o método "Tropical Soil Biology and Fertility". Utilizaram-se, ainda, amostras de solo indeformadas para avaliação dos atributos físicos do solo (densidade do solo, porosidade total, macro e microporosidade). Entre os sistemas produtivos, a melhor estrutura do solo foi verificada no sistema ILP, nas camadas de $0-0,1$ e $0,1-0,2 \mathrm{~m}$. Os sistemas sob ILP e em pastagem cultivada continuamente favoreceram um ambiente edáfico biologicamente mais ativo em comparação aos outros sistemas cultivados. A visualização conjunta dos atributos do solo mostrou que a integração lavoura-pecuária é uma estratégia para desenvolver sistemas de produção sustentáveis e que o período de dois anos de manejo é adequado para a rotação lavoura-pecuária.
\end{abstract}

Termos para indexação: diversidade, estrutura do solo, macrofauna do solo, perfil cultural.

\section{Joint analysis of physical and biological soil attributes under crop-livestock integration system}

\begin{abstract}
The objective of this work was to evaluate the effects of the integrated crop-livestock system on the morphological, physical and biological properties of soil. The experiment was carried out in Dourados, Mato Grosso do Sul State, Brazil, in a typic Hapludox soil. The following systems were sampled: grain crop under conventional soil tillage system, integrated crop-livestock system under no-tillage, continuous Urochloa decumbens (Syn. Brachiaria decumbens) pasture and natural system. In each system, a trench was opened in order to detect morphological changes caused by different management, using the cultural profile method. Assessments of soil macroinvertebrates were done according to the method Tropical Soil Biology and Fertility. Undisturbed soil samples were also used to assess the physical attributes of soil (soil density, total porosity, macro and microporosity). Among the cultivated systems, better soil structure was observed in the integrated crop-livestock system, in the layers $0-0.1$ and $0.1-0.2 \mathrm{~m}$. The systems under crop-livestock and pasture continuously cultivated favored a more biologically active soil environment, among the other agricultural systems. The visualization of soil attributes showed that crop-livestock integration is a promising strategy to develop sustainable production systems and that the period of two years of management is considered suitable for crop-pasture rotation.
\end{abstract}

Index terms: diversity, soil structure, soil macrofauna, crop profile.

\section{Introdução}

A exploração intensiva da pastagem deflagra processos de exaustão e degradação dos solos, o que ocasiona a redução da capacidade produtiva da unidade pecuária (Oliveira et al., 2004). Na atualidade, o grande número de áreas de pastagem com baixa capacidade produtiva, tem resultado em desafio para os órgãos de pesquisa e extensão, no sentido de viabilizar sistemas de produção que possibilitem maior eficiência energética e conservação ambiental (Macedo, 2009). Neste contexto, demonstra-se que a rotação de lavouras e pastagens em sistema plantio direto (SPD), em função de inúmeros e inquestionáveis benefícios, constitui estratégia viável técnica e economicamente para a recuperação e renovação de pastagens degradadas

Pesq. agropec. bras., Brasília, v.46, n.10, p.1277-1283, out. 2011 
(Vilela et al., 2003), o que gera alternativas de lucro e uma nova dinâmica do agronegócio local e regional (Freitas, 2002).

Diversos autores enfatizam que o sistema integrado lavoura-pecuária tem potencial para aumentar a produtividade e reduzir os riscos de degradação, ao melhorar as propriedades químicas, físicas e biológicas e o potencial produtivo do solo (Aidar \& Kluthcouski, 2003; Balbinot Junior et al., 2009; Macedo, 2009). As pastagens, de modo geral, têm a capacidade de manter ou até mesmo aumentar o teor de matéria orgânica (MO) do solo, em contraste com os cultivos anuais (Vilela et al., 2003), e são favorecidas pela grande quantidade de resíduos de material orgânico e pelo sistema radicular extenso e em constante renovação. Esses resíduos, além de diminuir a amplitude térmica e conservar melhor a água no solo, são fator determinante da redução da erosão hídrica (Hernani et al., 2002), da estabilização dos agregados, da aeração do solo (Castro Filho et al., 1998), e da diversidade dos organismos do solo (Silva et al., 2008a), os quais desempenham importantes atividades como a decomposição, a ciclagem de nutrientes, a síntese e a mineralização da MO no solo (Decaëns et al., 2003).

A relação entre o manejo e a qualidade do solo pode ser avaliada pelo efeito nas propriedades físicas, químicas e biológicas do solo (Doran \& Parkin, 1994). O entendimento da interação entre essas propriedades é fundamental para nortear as atividades antrópicas, que visam a utilização mais racional do ecossistema, em especial aqueles associados ao manejo dos solos no contexto de produção agrícola sustentável.

O método do perfil cultural do solo utilizado no Brasil desde a década de 1990, permite destacar diferentes unidades morfológicas no perfil dos sistemas, conforme o manejo adotado (Pereira Neto et al., 2007). Este método possibilita o entendimento e, consequentemente, a identificação de manejos mais adequados, a partir da análise das interações biológicas, físicas e químicas do solo (Tavares Filho et al., 1999).

O objetivo deste trabalho foi avaliar o efeito do sistema integração lavoura-pecuária sobre os atributos morfológicos, físicos e biológicos do solo.

\section{Material e Métodos}

O estudo foi realizado em julho do ano de 2003, num experimento estabelecido em 1995 na Embrapa
Agropecuária Oeste, em Dourados, MS (22¹4'00"S e $\left.54^{\circ} 49^{\prime} 00^{\prime \prime} \mathrm{W}\right)$, em Latossolo Vermelho distroférrico típico, com $700 \mathrm{~g} \mathrm{~kg}^{-1}$ de argila, em relevo plano com declive até $3 \%$. O clima da região, segundo a classificação de Köppen, é do tipo Aw, com estação quente e chuvosa no verão e moderadamente seca no inverno (Amaral et al., 2000).

Os tratamentos foram dispostos em faixas sob três sistemas de produção descritos a seguir. O sistema convencional (SC) de preparo do solo consistiu-se de cultivo de soja (Glycine max (L.) Merril) no verão e de aveia (Avena strigosa Schreb) no outono/inverno. O solo foi preparado com grades de disco até $0,2 \mathrm{~m}$ de profundidade (uma gradagem pesada e duas gradagens médias) com a utilização de herbicida residual em pré-emergência numa área de 2,0 ha. Também foi utilizado o sistema integração lavoura-pecuária (ILP) com faixa subdividida em duas partes de 4,0 ha, das quais uma ocupada com lavoura (ILP-L) e outra com pastagem (ILP-P), de forma a conter as duas fases da rotação no mesmo momento. Esse sistema é rotacionado a cada dois anos, quando a área ocupada com lavoura passa a ser ocupada com pastagem de Urochloa decumbens (Stapf) R.D.Webster (Syn. Brachiaria decumbens Stapf) e vice-versa. $\mathrm{Na}$ área ocupada com lavoura, a soja é manejada em SPD e, no inverno, é sucedida pela aveia, utilizada como pastagem temporária. Os animais foram manejados (carga de duas unidades animais (UA) de $450 \mathrm{~kg}$ por ha) em pastejo rotacionado sem suplementação no período da seca, com todas as recomendações técnicas sanitárias. Além dos dois sistemas descritos, utilizou-se a pastagem contínua (PC), um sistema com pastagem permanente de $U$. decumbens conduzida via pastejo rotacionado, com $2 \mathrm{UA} \mathrm{ha}^{-1}$, numa área de cerca de 10 ha. Uma área adjacente com vegetação nativa (VN) do tipo Floresta Estacional Semidecídua com cerca de 5 ha foi incluída, como referencial da condição original do solo.

Em cada área, foi aberta uma trincheira de 2,0 m de comprimento por 1,0 m de largura e 1,0 m de profundidade, perpendicular ao sentido da operação dos implementos, com vistas a detectar as modificações morfológicas provocadas pelos diferentes manejos. Utilizou-se o método do perfil cultural (Tavares Filho et al., 1999).

Os macroinvertebrados do solo foram amostrados em 5 pontos equidistantes de $30 \mathrm{~m}$ em monólitos $(0,25 \times 0,25 \mathrm{~m}$ de largura e $0,3 \mathrm{~m}$ de profundidade $)$ 
divididos em camadas de $0-0,1,0,1-0,2$ e $0,2-0,3 \mathrm{~m}$, conforme o método "Tropical Soil Biology and Fertility" (Anderson \& Ingram, 1993; Bignell, 2009). Após a extração manual dos macroinvertebrados, procedeu-se à identificação e contagem de grandes grupos taxonômicos, com auxílio de lupa binocular. A comunidade da macrofauna invertebrada edáfica foi avaliada por meio de densidade (indivíduos $\mathrm{m}^{-2}$ ) e riqueza (número de grupos). Nos mesmos pontos foram retiradas amostras de solo indeformadas, nas camadas de $0-0,1,0,1-0,2$ e $0,2-0,3 \mathrm{~m}$, as quais foram analisadas no Laboratório de Solo da Embrapa Agropecuária Oeste, para a avaliação dos atributos físicos (densidade do solo, porosidade total, macro e microporosidade) e teor de matéria orgânica do solo (MO), de acordo com Claessen (1997).

Os dados de densidade de indivíduos da macrofauna do solo, com base na sua heterogeneidade, foram transformados em $(x+0,5)^{0,5}$. Os resultados foram submetidos à análise de variância, com uso do delineamento inteiramente casualizado, e as médias foram comparadas pelo teste de Duncan, a $5 \%$ de probabilidade. Além disso, procedeu-se à análise de agrupamento, e adotou-se o método do vizinho mais distante ("complete linkage"), a partir da distância euclidiana, para descrever a similaridade entre os sistemas estudados. Nesta analise, foram consideradas as médias dos teores de $\mathrm{MO}$, macrofauna invertebrada edáfica e propriedades físicas do solo. As análises estatísticas foram processadas com o programa Statistica (StatSoft, 2005).

\section{Resultados e Discussão}

As estruturas observadas no perfil cultural da vegetação nativa destacou-se pela camada de 0,03 a 0,2 $\mathrm{m}$, que mostrou um volume de solo $(\mathrm{F} \mu \Delta / \mu)$ fissurado (F), formado por torrões subangulares médios (mt) e pequenos (pt), com alta porosidade interna visível $(\mu)$ e algumas porções em processo de compactação $(\mu \Delta)$, e alta atividade biológica, caracterizada pelo amontoamento de pequenos agregados (Figura 1). No sistema convencional, havia uma camada compacta de 0,07 a $0,24 \mathrm{~m}$, formada por grandes torrões compactos $(\Delta)$ e em processo de compactação $(\Delta \mu)$, com tendência a formas prismáticas e com fissuras bem visíveis (F), e baixa atividade biológica. Essa estrutura de solo é uma propriedade considerada como resultante negativa do uso intensivo de máquinas agrícolas no preparo do solo. Na pastagem contínua, havia uma camada de 0,04 a $0,17 \mathrm{~m}$ composta por grandes torrões compactos $(\Delta)$ e em processo de compactação $(\Delta \mu)$, com amontoamento de microagregados nas fissuras, que resulta, provavelmente, da presença de atividade biológica. Essa camada iniciava-se em uma base horizontal, formada por uma camada laminar compacta de $0,02 \mathrm{~m}$, que pode causar redução na infiltração de água no solo.

No sistema de integração lavoura-pecuária, na área com lavoura (ILP-L), havia uma camada superficial de 0-0,1 m, formada por pequenos torrões (pt) em processo de compactação $(\Delta \mu)$ e agregados resultantes da atividade de minhocas (coprólitos). Em seguida, uma camada de 0,1 a $0,2 \mathrm{~m}$ com agregados maiores e mais compactos $(\Delta)$ e presença de canais biológicos de vários tamanhos, formada por ação de macroinvertebrados do solo. Com relação à estrutura da camada superficial de 0 a $0,03 \mathrm{~m}$ da área com pastagem (ILP-P), houve a formação de uma camada laminar compacta $(\mathrm{Z} \Delta)$, composta por pequenos torrões (pt) compactos $(\Delta)$. Segundo Lanzanova et al. (2007), os efeitos da compactação pelo pisoteio animal concentram-se na camada superficial do solo. Na sequência, havia uma camada de 0,03 a 0,23 m fissurada, composta de pequenos e médios agregados compactos $(\Delta)$ e em processo de compactação $(\Delta \mu)$, com forte rugosidade e porosidade. Notou-se a presença de bioporos em todo perfil, por ação de macroinvertebrados do solo, que favorecem a penetração das raízes. As propriedades morfológicas do solo, observadas no sistema integrado, demonstram que, após o período de dois anos, o manejo pode ser considerado adequado para a rotação do sistema de integração lavoura-pecuária.

$\mathrm{Na}$ comparação entre os sistemas de produção, os valores de densidade do solo foram similares (Tabela 1). Cabe ressaltar que, na camada de 0 a $0,1 \mathrm{~m}$, a vegetação nativa, que representa a condição estrutural original do solo, teve menor densidade do solo em relação aos sistemas de produção nas três camadas avaliadas, com exceção do sistema pastagem contínua, na camada de 0 a $0,1 \mathrm{~m}$.

$\mathrm{O}$ volume de macroporos não foi afetado pelos diferentes sistemas de produção nas três camadas avaliadas, exceto na camada de 0 a $0,2 \mathrm{~m}$, na qual a vegetação natural apresentou maior macroporosidade (Tabela 1). Resultados semelhantes foram obtidos por Schaefer et al. (2001) e Falleiro et al. (2003). Quanto 
ao volume de microporos, não foram observadas diferenças entres os sistemas de produção na camada de 0 a $0,1 \mathrm{~m}$. Em estudos desenvolvidos por Spera et al. (2004) sobre sistemas de produção de grãos envolvendo pastagem sob SPD, em Latossolo Vermelho distrófico típico, verificaram-se diferenças de microporosidade entre os sistemas de manejo. Na camada de 0,1 a $0,2 \mathrm{~m}$, o ILP-P mostrou maior volume de microporos do que o $\mathrm{SC}$, enquanto que na camada de 0,2 a $0,3 \mathrm{~m}$, os valores de microporos do ILP-P foram maiores que os do SC.

Não houve diferenças entre os sistemas de produção nas camadas de 0 a 0,1 e de 0,2 a $0,3 \mathrm{~m}$, no que se refere à porosidade total (VTP), (Tabela 1). Na camada de 0,1 a 0,2 m, o ILP-L mostrou maior VTP que o SC. A VN mostrou maior porosidade total em relação aos sistemas cultivados, em todas as camadas, o que ressaltou a importância dos resíduos vegetais na estruturação dos solos (Spera et al., 2004).

A matéria orgânica do solo (MO) apresentou teores maiores sob VN, em virtude da maior diversidade de espécies vegetais (Silva et al., 2008b). Entre os sistemas de produção, o PC mostrou, nas camadas de 0 a 0,1 e 0,1 a $0,2 \mathrm{~m}$, maior valor de $\mathrm{MO}$ em relação ao SC. Amanutenção dos teores mais elevados de MO decorre do acúmulo de resíduos vegetais sobre a superfície do solo, do sistema radicular extenso e em constante renovação das pastagens,
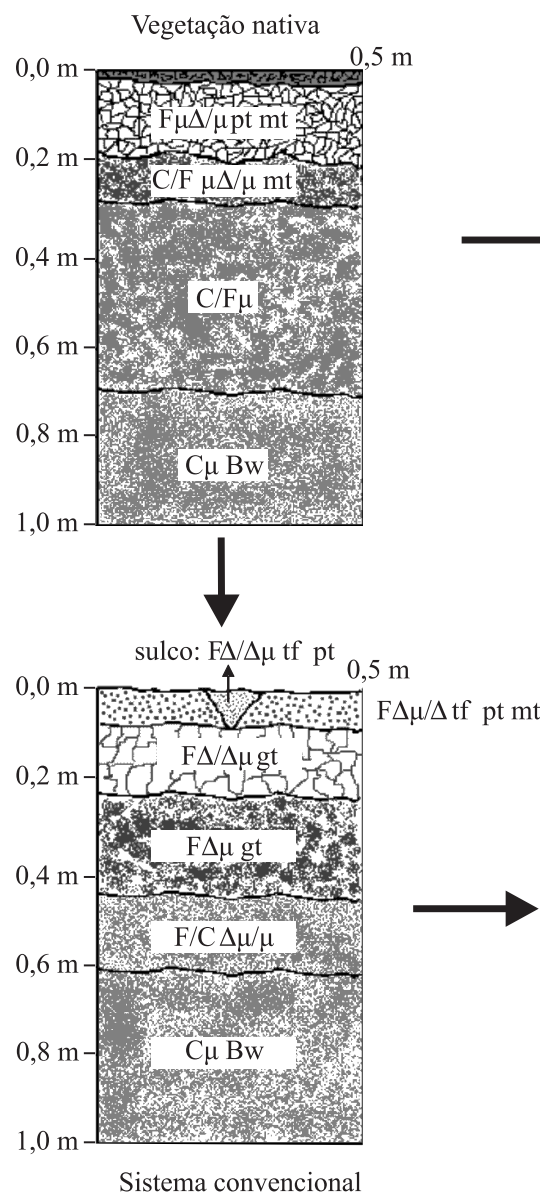
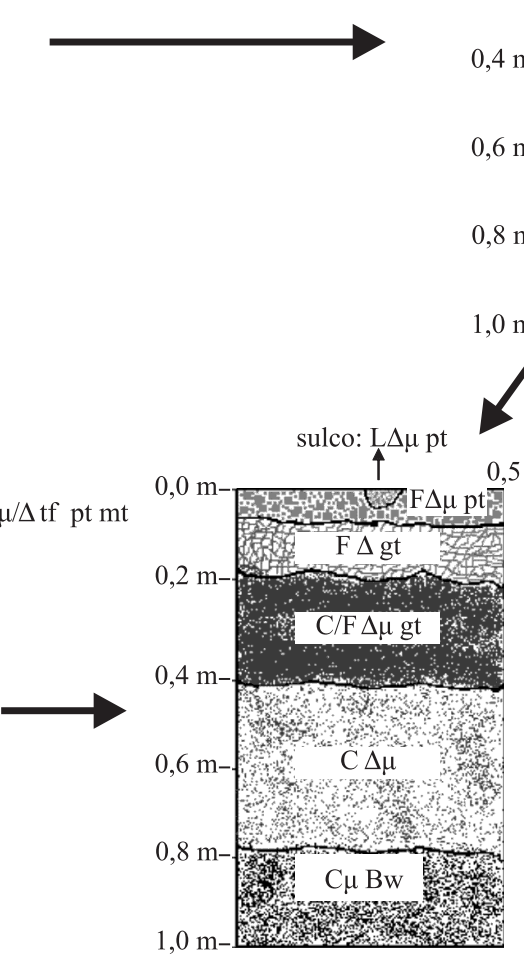

ILP-L ( 2 anos)

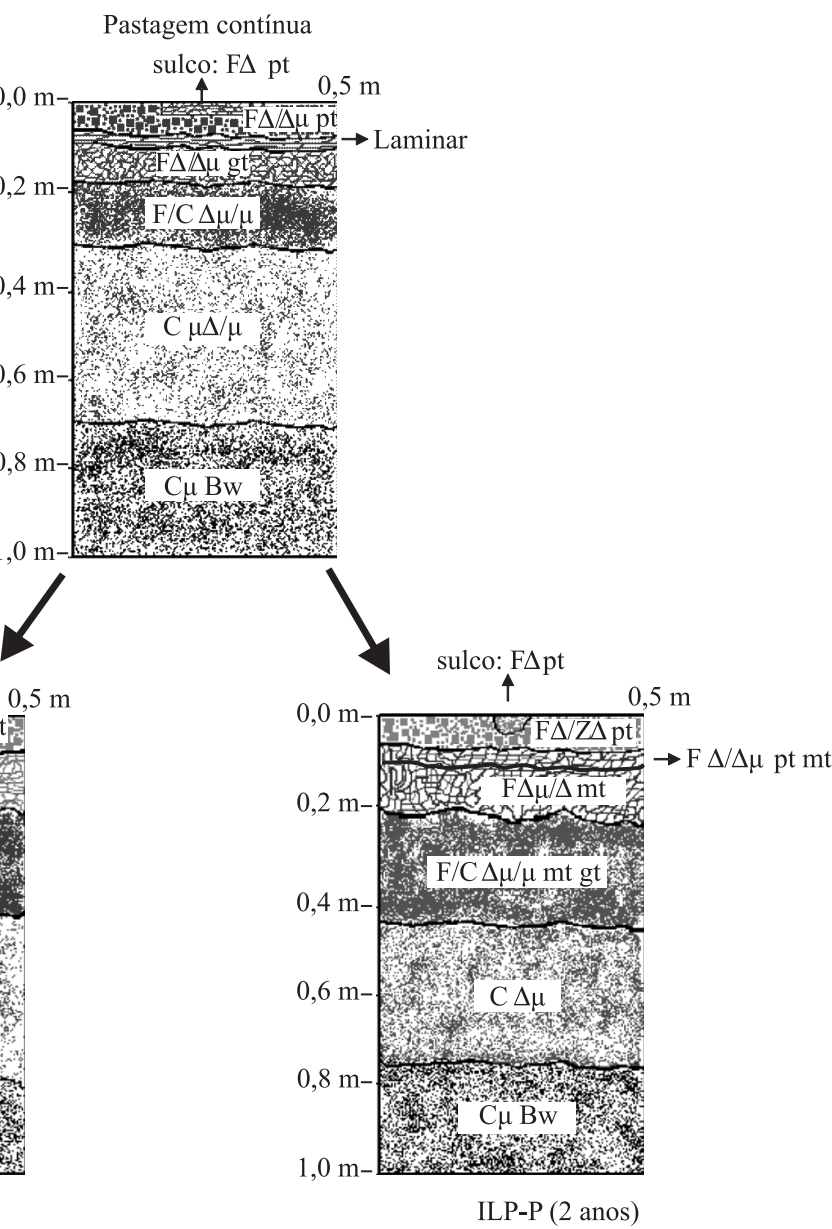

Figura 1. Representação esquemática das diferentes organizações dos perfis culturais, sob vegetação nativa, sistema convencional de preparo do solo, pastagem contínua e integração lavoura-pecuária (ILP). As setas indicam as modificações observadas a partir da vegetação nativa. L, volume de solo solto, poroso, constituído de torrões de tamanhos variados; F, volume de solo formado de agregados separados por uma rede de fissuras; $\mathrm{C}$, volume de solo contínuo, em que não se distinguem agregados; $\mu$, torrões com forte porosidade interna, principalmente arredondada; $\Delta$, torrões compactos, com baixa ou nenhuma porosidade visível; $\mu \Delta$ e $\Delta \mu$, porosidade interna dos torrões intermediária; (Z $\Delta$ ), camada laminar compacta; tf, terra fina; mt, médios torrões; gt, grandes torrões; pt, pequenos torrões, resultantes de atividade biológica; Bw, horizonte encontrado nos Latossolos $(\mathrm{C} \mu)$. 
e de dejetos de animais, os quais têm importante papel na ciclagem de nutrientes (Vilela et al., 2003). Na camada de 0,2 a 0,3 m, o teor de MO não diferiu entre os sistemas de produção, apenas da vegetação nativa.

A densidade e riqueza de grupos (diversidade) da macrofauna invertebrada do solo mostraram diferenças entre os sistemas estudados (Tabela 2). O SC e ILP-L tiveram menores valores de densidade nas camadas de 0 a 0,1 e de 0,1 a 0,2 m em comparação aos demais sistemas, o que denota que a macrofauna edáfica é altamente sensível a interferências antrópicas; portanto, o tipo de manejo e as práticas culturais determinam a presença e a quantidade de macroinvertebrados nos solos (Silva et al., 2008a; Marchão et al., 2009). Na camada de 0,2-0,3 m, os menores valores de densidade foram observados no SC e na VN.

Quanto à densidade total $(0-0,3 \mathrm{~m})$, o $\mathrm{SC}$ mostrou o menor valor em relação aos outros sistemas, o que, provavelmente, pode ser atribuído às modificações impostas pela aração e gradagem, tais como, a destruição do habitat, a exposição aos predadores, a eliminação do alimento disponível e as condições edafoclimáticas desfavoráveis (Decaëns et al., 2003; Aquino et al., 2008; Silva et al., 2008a). O contrário ocorre no sistema de integração lavoura-pecuária (ILP-L e ILP-P). Além do não revolvimento do solo, há maior deposição de resíduos vegetais, com a rotação de culturas e a intensa renovação da massa radicular das pastagens, o que resulta em mais exsudatos radiculares, em mudanças menos drásticas de temperatura e de conteúdo de água no solo, e no aumento de teor de MO, o que cria um microclima com atributos edafoclimáticos favoráveis à macrofauna invertebrada do solo (Sánchez \& Crespo, 2004; Silva et al., 2008a).

A densidade total da macrofauna edáfica no ILP-P e PC foi semelhante à da $\mathrm{VN}$, mas com menor riqueza de grupos, com 9 e 15 grupos taxonômicos, respectivamente. O SC mostrou valor de riqueza de grupos semelhante ao ILP-P e ILP-L, e inferior aos outros sistemas. A diversidade dos invertebrados, neste sistema de produção, desempenha papel importante nos processos biogeoquímicos do solo, pois a maior parte da energia absorvida pelas plantas é utilizada pela biota do solo para uma série de funções essenciais ao funcionamento do sistema (Silva et al., 2008a). Estas funções incluem a decomposição, a ciclagem de nutrientes, a mineralização da MOS, a modificação da estrutura do solo, a regulação da composição atmosférica e o controle biológico de pragas e doenças (Sánchez \& Reyes, 2003).

$\mathrm{Na}$ análise de agrupamento, técnica cujo objetivo é agrupar sistemas de manejo com base em características comuns, observou-se a formação de dois grandes grupos distintos com relação aos teores de MO, macrofauna invertebrada edáfica e propriedades

Tabela 1. Caracterização dos atributos físicos de solo, nas camadas de $0-0,1 \mathrm{~m}$, de $0,1-0,2 \mathrm{~m}$ e de $0,2-0,3 \mathrm{~m}$, sob sistema convencional de preparo do solo (SC), integração lavoura-pecuária com lavoura (ILP-L) e com pastagem (ILP-P), pastagem contínua (PC) e vegetação nativa $(\mathrm{VN})^{(1)}$.

\begin{tabular}{|c|c|c|c|c|c|}
\hline Manejo & $\begin{array}{c}\text { Ds } \\
\left(\mathrm{Mg} \mathrm{m}^{-3}\right) \\
\end{array}$ & Macroporos & $\begin{array}{l}\text { Microporos } \\
---\left(\mathrm{m}^{3} \mathrm{~m}^{-3}\right)-\end{array}$ & $\begin{array}{l}\text { VTP } \\
-\end{array}$ & $\begin{array}{c}\mathrm{MO} \\
\left(\mathrm{g} \mathrm{kg}^{-1}\right) \\
\end{array}$ \\
\hline & & & $0-0,1 \mathrm{~m}$ & & \\
\hline $\mathrm{SC}$ & $1,31 \mathrm{a}$ & $0,151 \mathrm{a}$ & $0,420 \mathrm{a}$ & $0,571 \mathrm{~b}$ & $26,6 \mathrm{c}$ \\
\hline ILP-P & $1,36 \mathrm{a}$ & $0,124 \mathrm{a}$ & $0,435 \mathrm{a}$ & $0,560 \mathrm{~b}$ & $32,0 \mathrm{bc}$ \\
\hline ILP-L & $1,38 \mathrm{a}$ & $0,148 \mathrm{a}$ & $0,431 \mathrm{a}$ & $0,580 \mathrm{~b}$ & $32,1 \mathrm{bc}$ \\
\hline PC & $1,25 \mathrm{ab}$ & $0,151 \mathrm{a}$ & $0,416 \mathrm{a}$ & $0,567 \mathrm{~b}$ & $35,8 \mathrm{~b}$ \\
\hline \multirow[t]{2}{*}{$\underline{\mathrm{VN}}$} & $1,17 \mathrm{~b}$ & $0,183 \mathrm{a}$ & $0,444 \mathrm{a}$ & $0,627 \mathrm{a}$ & $67,6 \mathrm{a}$ \\
\hline & & & $0,1-0,2 \mathrm{~m}$ & & \\
\hline $\mathrm{SC}$ & $1,42 \mathrm{a}$ & $0,106 \mathrm{~b}$ & $0,421 \mathrm{c}$ & $0,526 \mathrm{c}$ & $23,3 \mathrm{c}$ \\
\hline ILP-P & $1,41 \mathrm{a}$ & $0,095 b$ & $0,460 \mathrm{a}$ & $0,555 \mathrm{bc}$ & $23,5 \mathrm{c}$ \\
\hline ILP-L & $1,39 \mathrm{a}$ & $0,116 b$ & $0,449 \mathrm{ab}$ & $0,565 \mathrm{~b}$ & $26,3 b c$ \\
\hline $\mathrm{PC}$ & $1,33 \mathrm{a}$ & $0,122 b$ & $0,435 \mathrm{bc}$ & $0,557 \mathrm{bc}$ & $30,6 b$ \\
\hline \multirow[t]{2}{*}{$\mathrm{VN}$} & $1,18 \mathrm{~b}$ & $0,176 \mathrm{a}$ & $0,442 \mathrm{abc}$ & $0,617 \mathrm{a}$ & $41,4 \mathrm{a}$ \\
\hline & & & $0,2-0,3 \mathrm{~m}$ & & \\
\hline $\mathrm{SC}$ & $1,47 \mathrm{a}$ & $0,096 \mathrm{a}$ & $0,426 \mathrm{~b}$ & $0,523 a$ & $21,2 \mathrm{~b}$ \\
\hline ILP-P & $1,42 \mathrm{a}$ & $0,102 \mathrm{a}$ & $0,451 \mathrm{a}$ & $0,552 \mathrm{a}$ & $20,5 b$ \\
\hline ILP-L & $1,41 \mathrm{a}$ & $0,108 \mathrm{a}$ & $0,439 \mathrm{ab}$ & $0,547 \mathrm{a}$ & $28,1 \mathrm{ab}$ \\
\hline $\mathrm{PC}$ & $1,42 \mathrm{a}$ & $0,111 \mathrm{a}$ & $0,444 \mathrm{ab}$ & $0,555 \mathrm{a}$ & $25,0 \mathrm{ab}$ \\
\hline $\mathrm{VN}$ & $1,18 \mathrm{~b}$ & $0,166 \mathrm{a}$ & $0,431 \mathrm{ab}$ & $0,597 \mathrm{a}$ & $31,1 \mathrm{a}$ \\
\hline
\end{tabular}


físicas do solo (Figura 2). Esses dois grupos não apresentaram nenhuma similaridade entre si, uma vez que a sua distância de ligação foi de $100 \%$. No primeiro subgrupo, ocorreu a formação de dois agrupamentos (subgrupos) distintos. O primeiro subgrupo mostrou uma ligação com distância inferior a 5\% entre o VN e $\mathrm{PC}$, na profundidade de $0-0,1 \mathrm{~m}$, o que permite inferir que a similaridade entre eles é de 95\%. Possivelmente, o agrupamento ocorreu em virtude da alta abundância de cupins ( $75 \%$ da densidade total) na pastagem contínua. O tipo de manejo do solo pode influenciar a abundância de indivíduos e a predominância de grupos em determinado sistema (Barros et al., 2002).

Tabela 2. Macrofauna invertebrada do solo sob sistema convencional de preparo do solo (SC), integração lavourapecuária com lavoura(ILP-L) e com pastagem(ILP-P), pastagem contínua (PC) e vegetação nativa $(\mathrm{VN})^{(1)}$.

\begin{tabular}{|c|c|c|c|c|c|}
\hline \multirow[t]{2}{*}{ Sistema } & \multicolumn{3}{|c|}{ Densidade (indivíduos $\mathrm{m}^{-2}$ ) } & \multirow[t]{2}{*}{ Total } & \multirow{2}{*}{$\begin{array}{c}\text { Diversidade } \\
\left(\mathrm{N}^{\circ} \text { de grupos }\right)\end{array}$} \\
\hline & $0-0,1 \mathrm{~m}$ & $0,1-0,2 \mathrm{~m}$ & $0,2-0,3 \mathrm{~m}$ & & \\
\hline$\overline{\mathrm{SC}}$ & $90 \mathrm{~b}$ & $35 b$ & $16 \mathrm{c}$ & $141 \mathrm{c}$ & $6 c$ \\
\hline ILP-P & $470 \mathrm{a}$ & $336 a$ & $272 b$ & $1.107 \mathrm{ab}$ & $9 \mathrm{c}$ \\
\hline ILP-L & $198 b$ & $38 b$ & $512 \mathrm{a}$ & $893 b$ & $13 b c$ \\
\hline $\mathrm{PC}$ & $813 \mathrm{a}$ & $218 \mathrm{a}$ & $240 b$ & $1.389 \mathrm{a}$ & $15 b$ \\
\hline $\mathrm{VN}$ & $797 \mathrm{a}$ & $410 \mathrm{a}$ & $54 \mathrm{c}$ & $1.386 \mathrm{a}$ & $19 \mathrm{a}$ \\
\hline
\end{tabular}

${ }^{(1)}$ Médias seguidas de letras iguais, nas colunas, não diferem entre si pelo teste de Duncan, a 5\% probabilidade. ILP-L, lavoura com aveia cultivada em sucessão a soja; ILP-P e PC, pastagem de Urochloa decumbens.

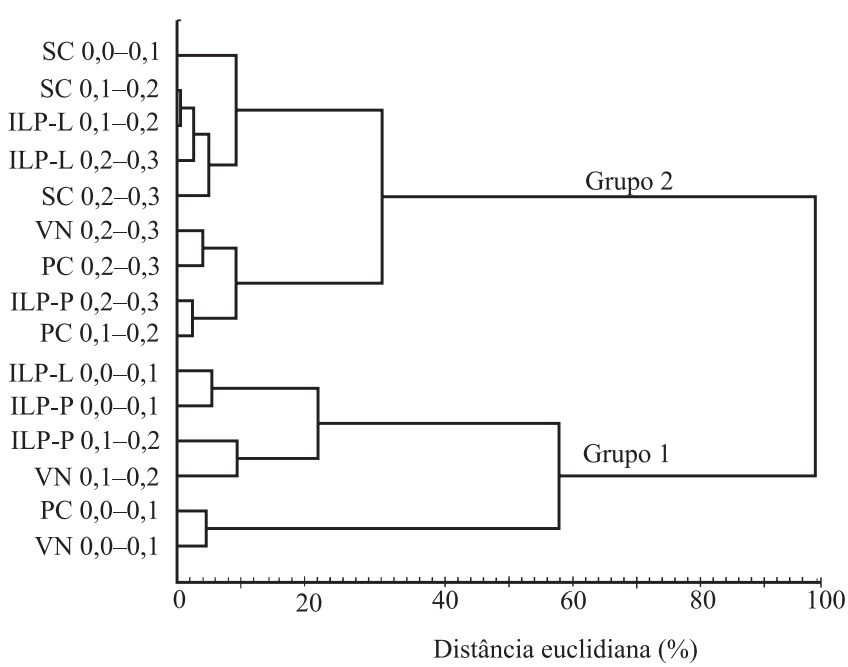

Figura 2. Dendrograma de similaridade, com bases nas distâncias euclidianas, das variáveis avaliadas entre os sistemas de manejo de solo nas profundidades de $0-0,1$, $0,1-0,2$ e $0,2-0,3 \mathrm{~m}$. Sistema convencional de preparo do solo (SC), integração lavoura-pecuária com lavoura (ILP-L) e com pastagem (ILP-P), pastagem contínua (PC) e vegetação nativa $(\mathrm{VN})$.
No segundo subgrupo, os sistemas VN (de 0,1-0,2 m), ILP-L (de $0-0,1 \mathrm{~m})$ e ILP-P $(0-0,1$ e $0,1-0,2 \mathrm{~m})$ apresentaram $78 \%$ de similaridade. Isto demonstra que o sistema integrado apresentou características semelhantes na fase lavoura e pastagem, na camada de 0-0,1 m, após o período de dois anos de manejo, o que indica que este período é adequado para a rotação do sistema lavoura-pecuária.

No segundo grupo, observou-se a formação de dois subgrupos de agrupamentos distintos. $\mathrm{O}$ primeiro subgrupo apresentou semelhança de $90 \%$ entre os sistemas VN, PC e ILP-P avaliados na profundidade de $0,2-0,3 \mathrm{~m}$, além do PC avaliado na camada $0,1-0,2 \mathrm{~m}$. A formação desse subgrupo demonstra que os efeitos dos sistemas de manejo desaparecem na profundidade de $0,2-0,3 \mathrm{~m}$. No segundo subgrupo, verificou-se que o $\mathrm{SC}$, cuja avaliação foi feita em todas as profundidades, apresentou $90 \%$ de similaridade com o ILP-P, avaliado nas profundidades de $0,1-0,2$ e de $0,2-0,3 \mathrm{~m}$. Possivelmente, o agrupamento ocorreu em virtude da redução da diversidade dos macroinvertebrados do solo.

\section{Conclusões}

1. A melhor estruturação do solo foi verificada no sistema de integração lavoura-pecuária, nas camadas mais superficiais do solo.

2. Os sistemas sob integração lavoura-pecuária e pastagem cultivada continuamente favorecem um ambiente edáfico biologicamente mais ativo, em comparação aos outros sistemas cultivados.

3. A integração lavoura-pecuária é uma estratégia promissora para desenvolver sistemas de produção sustentáveis, e o período de dois anos de manejo é adequado para a rotação lavoura-pecuária.

\section{Referências}

AIDAR, H.; KLUTHCOUSKI, J. Evolução das atividades lavoureira e pecuária nos cerrados. In: KLUSTHCOUSKI, J.; STONE, L.F.; AIDAR, H. (Ed.). Integração lavoura-pecuária. Santo Antônio de Goiás: Embrapa Arroz e Feijão, 2003. p.24-58.

AMARAL, J.A.M. do; MOTCHI, E.P.; OLIVEIRA, H. de; CARVALHO FILHO, A. de; NAIME, U.J.; SANTOS, R.D. dos. Levantamento semidetalhado dos solos do campo experimental de Dourados, da Embrapa Agropecuária Oeste, Município de Dourados, MS. Dourados: Embrapa Agropecuária Oeste; Rio de Janeiro: Embrapa Solos, 2000. 68p. (Embrapa Agropecuária Oeste. Documentos, 22; Embrapa Solos. Documentos, 15).

ANDERSON, J.M.; INGRAM, J.S.I. (Ed.). Tropical soil biology and fertility: a handbook of methods. 2.ed. Wallingford: $\mathrm{CAB}$, 1993. $221 \mathrm{p}$. 
AQUINO, A.M. de; SILVA, R.F. da; MERCANTE, F.M.; CORREIA, M.E.F.; GUIMARÃES, M. de F.; LAVELlE, P. Invertebrate soil macrofauna under different ground cover plants in the no-till system in the Cerrado. European Journal of Soil Biology, v.44, p.191-197, 2008.

BALBINOT JUNIOR, A.A.; MORAES, A. de; VEIGA, M. da; PELISSARI, A.; DIECKOW, J. Integração lavoura-pecuária: intensificação de uso de áreas agrícolas. Ciência Rural, v.39, p.1925-1933, 2009.

BARROS, E.; PASHANASI, B.; CONSTANTINO, R.; LAVELLE, P. Effects of land-use system on the soil macrofauna in western Brazilian Amazonia. Biology and Fertility of Soils, v.35, p.338-347, 2002.

BIGNELL, D.E. Towards a universal sampling protocol for soil biotas in the humid tropics. Pesquisa Agropecuária Brasileira, v.44, p.825-834, 2009.

CASTRO FILHO, C.; MUZILli, O.; PADANOSCHI, A.L. Estabilidade dos agregados e sua relação com o teor de carbono orgânico num Latossolo Roxo distrófico, em função de sistemas de plantio, rotações de culturas e métodos de preparo de amostras. Revista Brasileira de Ciência do Solo, v.22, p.527-538, 1998.

CLAESSEN, M.E.C. (Org.). Manual de métodos de análise de solo. 2.ed. rev. atual. Rio de Janeiro: Embrapa-CNPS, 1997. 212p. (Embrapa-CNPS. Documentos, 1).

DECAËNS, T.; LAVELLE, P.; JIMÉNEZ, J.J.; ESCOBAR, G.; RIPPSTEIN, G.; SCHNEIDMADL, J.; SANZ, J.I.; HOYOS, P.; THOMAS, R.J. Impacto del uso de la tierra en la macrofauna del suelo de los Llanos Orientales de Colombia. In: JIMÉNEZ, J.J.; THOMAS, R.J. (Ed.). El arado natural: las comunidades de macroinvertebrados del suelo en las sabanas neo tropicales de Colombia. Cali: Centro Internacional de Agricultura Tropical, 2003. p.21-45. (CIAT. Publicación, 336).

DORAN, J.W.; PARKIN, T.B. Defining and assessing soil quality. In: DORAN, J.W.; COLEMAN, D.C.; BEZDICEK, D.F.; ETEWART, B.A. (Ed.). Defining soil quality for a sustainable environment. Madison: Soil Science Society of America, 1994. p.3-21 (SSSA. Special publication, 35).

FALLEIRO, R.M.; SOUZA, C.M.; SILVA, C.S.W.; SEDIYAMA, C.S.; SILVA, A.A.; FAGUNDES, J.L. Influência dos sistemas de preparo nas propriedades químicas e físicas do solo. Revista Brasileira de Ciência do Solo, v.27, p.1097-1104, 2003.

FREITAS, P.L. Harmonia com a natureza: a adoção do sistema Plantio Direto pode garantir competitividade à agricultura brasileira, em especial na região dos cerrados. Agroanalysis, v.22, p.12-17, 2002.

HERNANI, L.C.; FREITAS, P.L. de; PRUSKI, F.F.; DE MARIA, I.C.; CASTRO FILHO, C. de; LANDERS, J.N. A erosão e seu impacto. In: MANZATO, C.V.; FREITAS JÚNIOR, E. de; PERES, J.R.R. (Ed.). Uso agrícola dos solos brasileiros. Rio de Janeiro: Embrapa Solos, 2002. p.47-60.

LANZANOVA, M.E.; NICOLOSO, R. da S.; LOVATO, T.; ELTZ, F.L.F.; AMADO, T.J.C.; REINERT, D.J. Atributos físicos do solo em sistema de integração lavoura-pecuária sob plantio direto. Revista Brasileira de Ciência do Solo, v.31, p.1131-1140, 2007.
MACEDO, M.C.M. Integração lavoura e pecuária: o estado da arte e inovações tecnológicas. Revista Brasileira de Zootecnia, v.38, p.133-146, 2009.

MARCHÃO, R.L.; LAVELLE, P.; CELINI, L.; BALBINO, L.C.; VILELA, L.; BECQUER, T. Soil macrofauna under integrated crop-livestock systems in a Brazilian Cerrado Ferralsol. Pesquisa Agropecuária Brasileira, v.44, p.1011-1020, 2009.

OLIVEIRA, O.C. de; OLIVEIRA, I.P. de; ALVES, B.J.R.; URQUIAGA, S.; BODDEY, R.M. Chemical and biological indicators of decline/degradation of Brachiaria pastures in the Brazilian Cerrado. Agriculture, Ecosystems and Environment, v.103, p.289-300, 2004.

PEREIRA NETO, O.C.; GUIMARÃES, M. de F.; RALISCH, R.; FONSECA, I.C.B. Análise do tempo de consolidação do sistema de plantio direto. Revista Brasileira de Engenharia Agrícola e Ambiental, v.11, p.489-496, 2007.

SÁNCHEZ, S.; CRESPO, G. Comportamiento de la macrofauna del suelo en pastizales con gramíneas puras o intercaladas con leucaena. Pastos y Forrajes, v.27, p.347-353, 2004.

SÁNCHEZ, S.; REYES, F. Estudio de la macrofauna edáfica en una asociación de Morus alba y leguminosas arbóreas. Pastos y Forrajes, v.26, p.315-320, 2003.

SCHAEFER, C.E.G.R.; SOUZA, C.M.; VALLEJOS M., F.J.; VIANA, J.H.M.; GALVÃO, J.C.C.; RIBEIRO, L.M. Características da porosidade de um Argissolo Vermelho-Amarelo submetido a diferentes sistemas de preparo de solo. Revista Brasileira de Ciência do Solo, v.25, p.765-769, 2001.

SILVA, R.F. da; AQUINO, A.M. de; MERCANTE, F.M.; GUIMARÃES, M. de F. Macrofauna invertebrada do solo em sistema integrado de produção agropecuária no Cerrado. Acta Scientiarum: Agronomy, v.30, p.725-731, 2008a.

SILVA, R.F. da; BORGES, C.D.; GARIB, D.M.; MERCANTE, F.M. Atributos físicos e teor de matéria orgânica na camada superficial de um Argissolo Vermelho cultivado com mandioca sob diferentes manejos. Revista Brasileira de Ciência do Solo, v.32, p.2435-2441, 2008b.

SPERA, S.T.; SANTOS, H.P.; FONTANELI, R.S.; TOMM, G.O. Efeitos de sistemas de produção de grãos envolvendo pastagens sob plantio direto nos atributos físicos de solo e na produtividade. Revista Brasileira de Ciência do Solo, v.28, p.533-542, 2004.

STATSOFT. Statistica for windows. Version 5.0. Tulsa: StatSoft, 1995.

TAVARES FILHO, J.; RALISCH, R.; GUIMARÃES, M.F.; MEDINA, C.C.; BALBINO, L.C.; NEVES, C.S.V.J. Método do perfil cultural para avaliação do estado físico de solos em condições tropicais. Revista Brasileira de Ciência do Solo, v.23, p.393-399, 1999.

VILELA, L.; MACEDO, M.C.M.; MARTHA JÚNIOR, G.B.; KLUTHCOUSKI, J. Benefícios da integração lavoura-pecuária. In: KLUTHCOUSKI, J.; STONE, L.F.; AIDAR, H. (Ed.). Integração lavoura-pecuária. Santo Antônio de Goiás: Embrapa Arroz e Feijão, 2003, p.145-170.

Recebido em 3 de julho de 2010 e aprovado em 8 de setembro de 2011 